\title{
IMPACTO DE LAS AGUAS SUPERFICIALES EN EL AUMENTO DEL NIVEL DE LAS AGUAS DEL LAGO ENRIQUILLO
}

\author{
Impact of surface waters on the increase \\ in the level of the waters of Lake Enriquillo
}

\section{Virgilio Antonio Miniño Mejía}

Instituto Tecnológico de Santo Domingo, INTEC, República Dominicana. Email: Virgilio.minino@intec.edu.do

\section{Yolanda León}

Instituto Tecnológico de Santo Domingo, INTEC, República Dominicana.

\section{Cándido Quintana}

Instituto Tecnológico de Santo Domingo, INTEC, República Dominicana.

Recibido: Enero 16, 2018. Aprobado: Febrero 27, 2018.

Cómo citar: Miniño Mejía, V. A., León, Y., \& Quintana, C. (2018). Impacto de las aguas superficiales en el aumento del nivel de las aguas del Lago Enriquillo. Ciencia, Ambiente Y Clima, 1(1), 9-21. https://doi.org/10.22206/cac.2018. v1i1.pp9-21

\section{Resumen}

A partir del 2014 el lago Enriquillo, localizado en el suroeste de la República Dominicana, empezó a evidenciar un continuo crecimiento en el nivel de sus aguas llegando, de $265 \mathrm{~km}^{2}$ hasta alrededor de $375 \mathrm{~km}^{2}$. Esto duró hasta el 2013 cuando se empezó a observar un decrecimiento paulatino hasta el momento. Durante todo este tiempo se han venido llevando a cabo investigaciones para determinar la causa, o causas, que dieron lugar a este fenómeno. Aunque se han planteado varias hipótesis sobre dicha crecida aún no existe un consenso generalizado sobre la crecida del lago. Lo que se plantea en este trabajo de investigación es que, exceptuando el aporte de aguas superficiales a través de eventos extremos, la mayor cantidad de agua que drena en el lago Enriquillo es a través del subsuelo, ya que la mayoría de los ríos y canales alrededor del lago han estado secos durante los últimos dos años. Gran parte del agua que fluye desde las sierras de Neyba y Bahoruco, desde el Norte y Sur respectivamente, es utilizada para riego y ganadería, en menor escala. El lago Enriquillo no recibe aporte de aguas superficiales desde la parte Oeste, y en cuanto al Este solo drena de manera continua el canal Cristóbal, el cual tiene un flujo que en gran medida depende del manejo de una compuerta y del uso para agricultura.

Palabras clave: Lago Enriquillo, sierra de Neyba, sierra de Bahoruco, agua superficial.

\begin{abstract}
Starting in 2014, Lake Enriquillo, located in the southwest of the Dominican Republic, began to show a continuous increase in the level of its waters, which lasted until 2013 when a gradual decrease started up to date. The change in water surface went from $265 \mathrm{~km}^{2}$ to approximately $375 \mathrm{~km} 2$. During all this time, investigations have been carried out to determine the cause, or causes, that gave rise to this phenomenon. Although several hypotheses have been raised about this phenomenon, there is still no widespread consensus on the causes of the rise in the lake's water. What is raised in this research is that, except for the contribution of surface water through extreme events, the largest amount of water that drains into Lake Enriquillo is through the subsoil, since most of the rivers and canals around the lake have been dry for the past two years. Much of the water flowing from the Neyba and Bahoruco mountain ranges, from the North and South respectively, is used for irrigation and livestock, on a smaller scale. Lake Enriquillo does not receive surface water from the west, and as far as the east, it only continuously drains water from Cristobal canal, which has a flow that largely depends on the handling of a dam and the use for agriculture.
\end{abstract}

Keywords: Enriquillo Lake, Neyba mountain range, Bahoruco mountain range, surface water. 


\section{Introducción}

El lago Enriquillo se encuentra en el suroeste de la República Dominicana en las coordenadas $18^{\circ} 30^{\prime} \mathrm{N} 71^{\circ} 35^{\prime} \mathrm{W}$ y una extensión de 3,319.51 $\mathrm{km}^{2}$, entre las sierras de Neyba y Bahoruco. Fig. 1. Es el más grande de las Antillas con una extensión superficial de aproximadamente $265 \mathrm{~km}^{2}$ (Mann et al. 1995, Koehler et al., 2009), siendo además un sistema endorreico que se encuentra a aproximadamente 40 metros bajo el nivel del mar, siendo el punto más bajo del Caribe. (Buck et al., 2005). La superficie del lago es de aproximadamente 265 $\mathrm{km}^{2}$, pero esta llegó a tener una extensión de más de $375 \mathrm{~km}^{2}$ en el año 2013. Después de esta fecha el lago empezó a evidenciar un decrecimiento en el nivel de sus aguas, pudiéndose observar un descenso de las aguas en sus costas, especialmente en el área descrita como la Azufrada. Figs. 2 y 3. La salinidad del lago por lo general es hipersalina, es decir mucho mayor que la salinidad del mar que es de aproximadamente $35 \mathrm{ppt}$, registrando salinidad tan alta como 110 ppt (Buck et al., 2005; INTEC \& CCNY 2013). Debido al aumento del nivel de sus aguas la salinidad del lago Enriquillo llegó a su nivel más bajo registrado, 22 ppt (Minińo et al., 2013). A partir del 2013, y en congruencia con el descenso del nivel de las aguas del lago, la salinidad ha ido aumentando hasta registrarse unas $39 \mathrm{ppt}$ en los primeros meses del año 2017. Esto debido a que el agua del lago se ha ido evaporando a un ritmo mayor en que ha recibido aguas dulces.

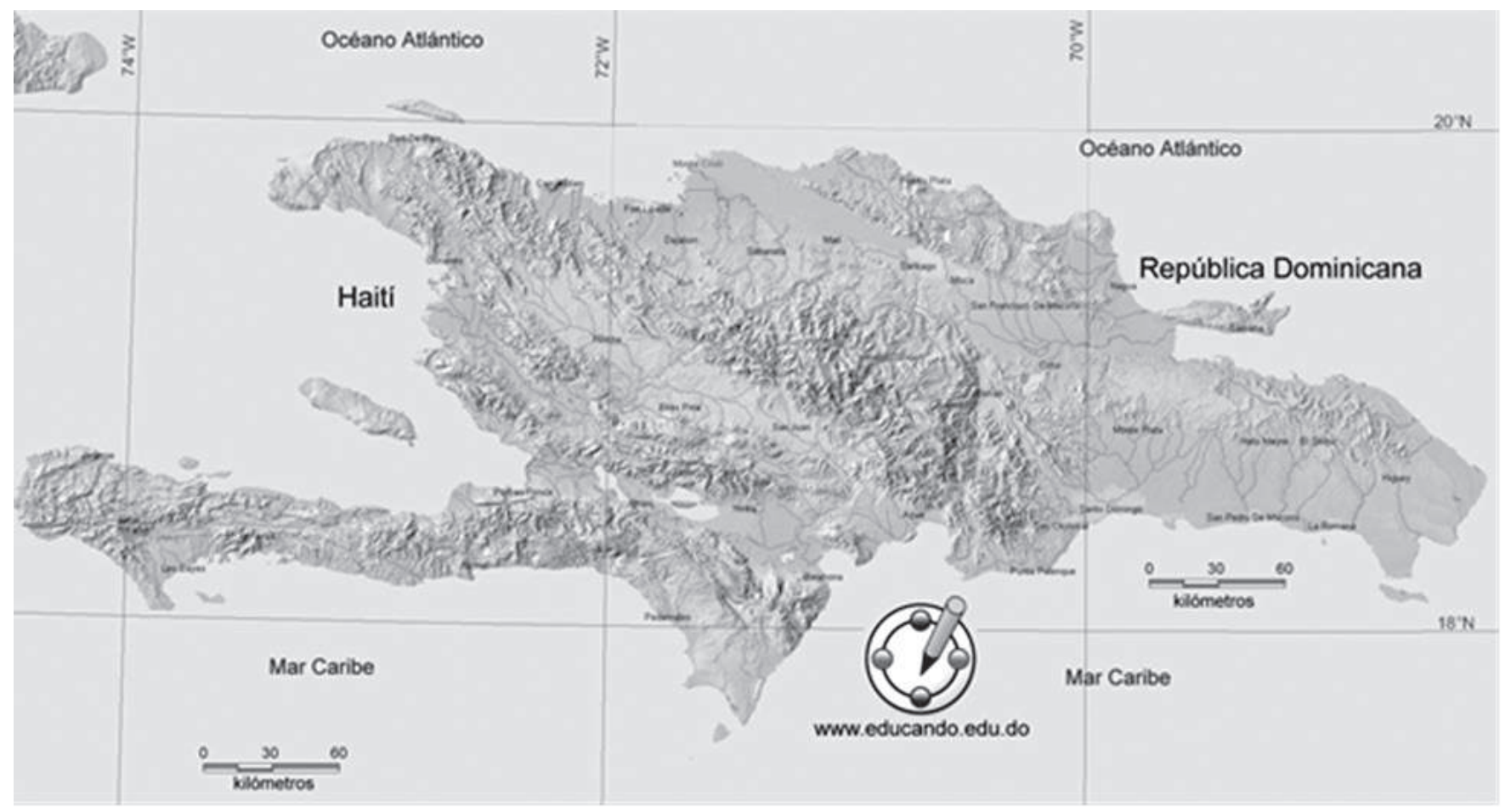

Figura 1. Mapa de la Española con la ubicación del lago Enriquillo. Fuente: www.educando.do 


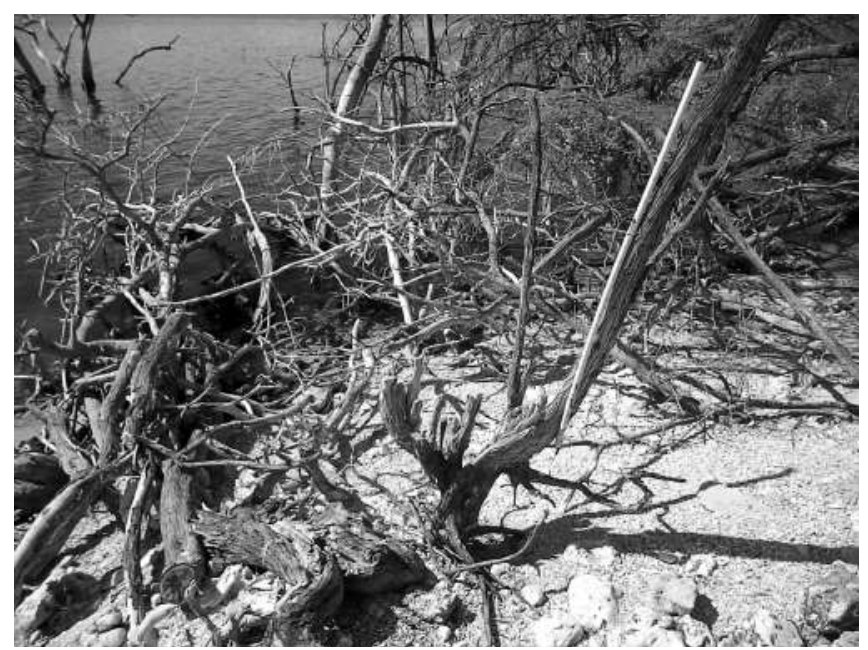

Figura 2. Nivel del lago Enriquillo en las costas de la Azufrada. 2014.

Desde que se empezó a evidenciar una crecida en el nivel de las aguas del lago Enriquillo se han realizado múltiples trabajos de investigación sobre las posibles causas de este fenómeno. Aún a la fecha no existe un consenso entre investigadores sobre la causa, o causas, del crecimiento del lago. Lo que sí ha quedado evidenciado es que este fenómeno tuvo una gran influencia de las tormentas Noel y Olga en el año 2007. (Cocco Quezada 2009; Martínez 2010; Payano \& Medrano, 2011; Romero et al., 2011; Miniño et al., 2015). Este trabajo de investigación aporta datos científicos sobre el aporte de las aguas superficiales a la crecida del lago Enriquillo, calculando y analizando el flujo de todas las fuentes alrededor del mismo.

\section{Geología del Lago Enriquillo}

El lago Enriquillo es el resultado de un canal marino que hace millones de años comunicaba la bahía de Neyba con Puerto Príncipe. (Mann 1991). Fig. 4. El lago se encuentra entre las sierras de Neyba y Bahoruco. Esta última está formada por un sistema de formaciones de rocas calizas cenozoicas, falladas y con formaciones cársticas, y con la cara norte separada de la Hoya del Lago Enriquillo por una escalera de fallas. (Eptisa, 2004, Mann et al., 1991).

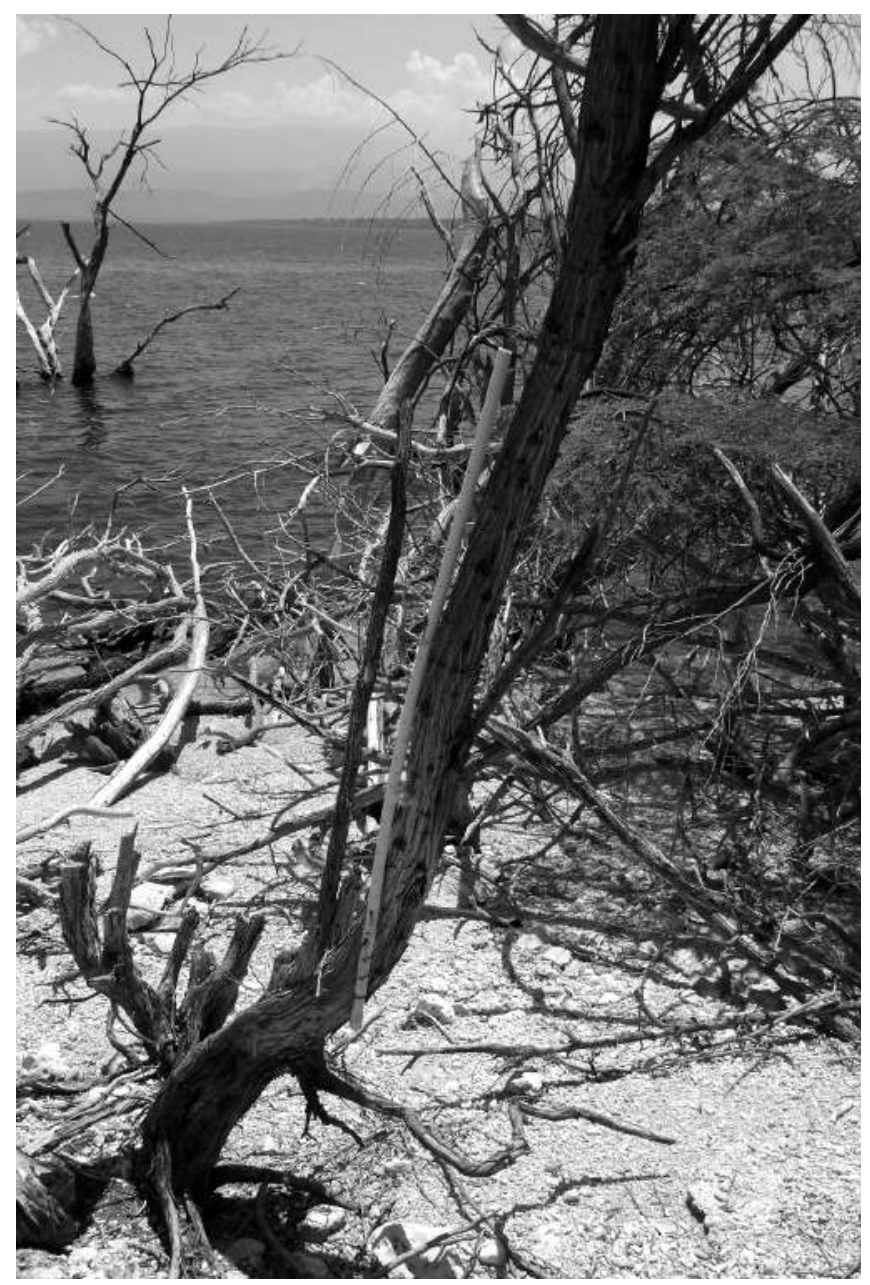

Figura 3. Nivel del lago Enriquillo en las costas de la Azufrada. 2016.

La geología de la sierra de Neyba también se caracteriza por un sistema de rocas calizas. Fig. 5. Esto permite que el agua subterránea fluya con facilidad hacia el lago Enriquillo, arrastrando también sales que contribuyen a la salinidad del mismo. El lago Enriquillo está ubicado en un valle formado por la falla Enriquillo, la cual que se extiende 127 millas desde la bahía de Puerto Príncipe en Haití en el oeste, hasta cerca de la bahía de Neiba en la República Dominicana en el este. Se le conoce como la Hoya de Enriquillo en la República Dominicana. (Dolan et al., 1991). Los temblores en la región son comunes, aunque generalmente por debajo de 3.0 en la escala de Richter. 


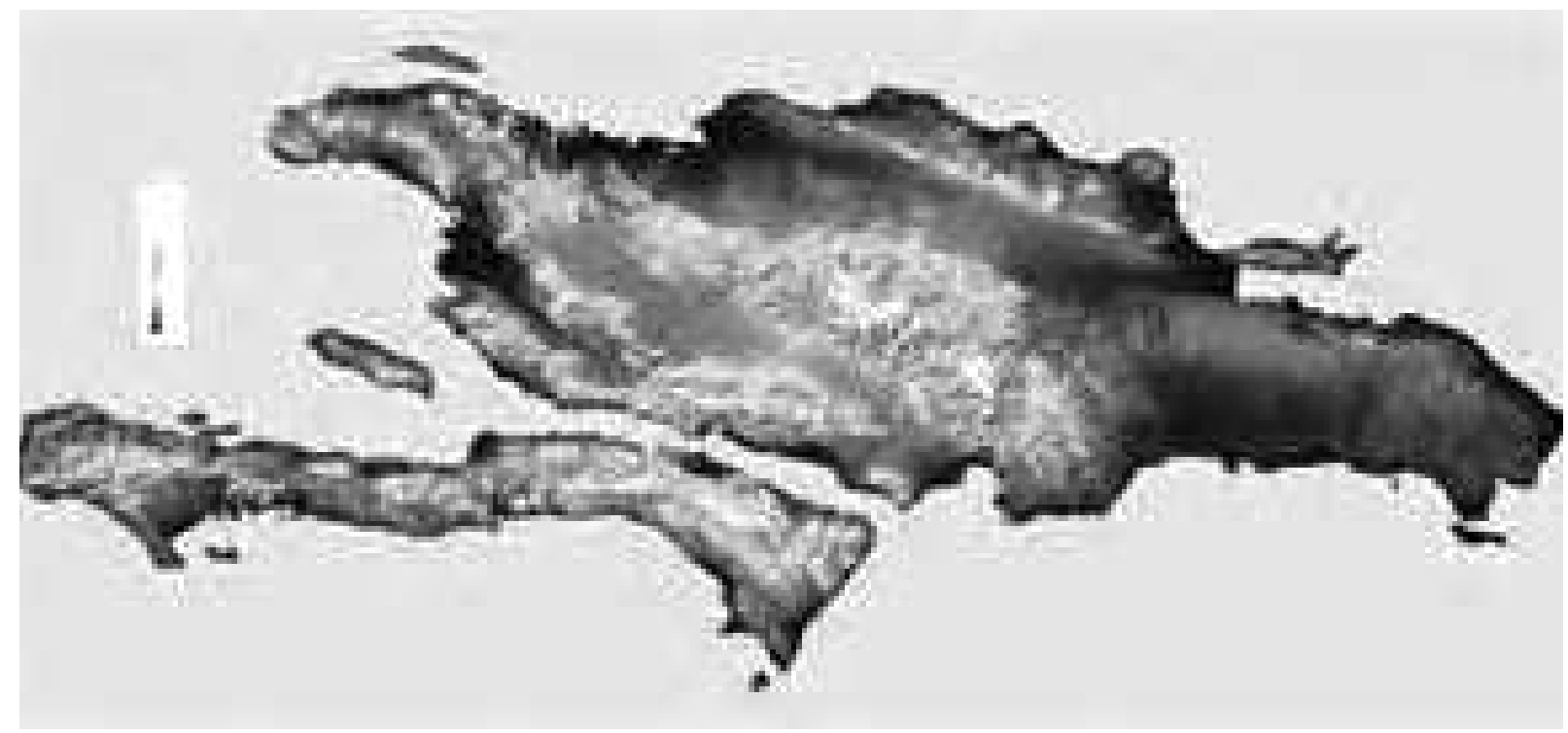

Figura 4. Canal marino en la Española. Fuente: Google

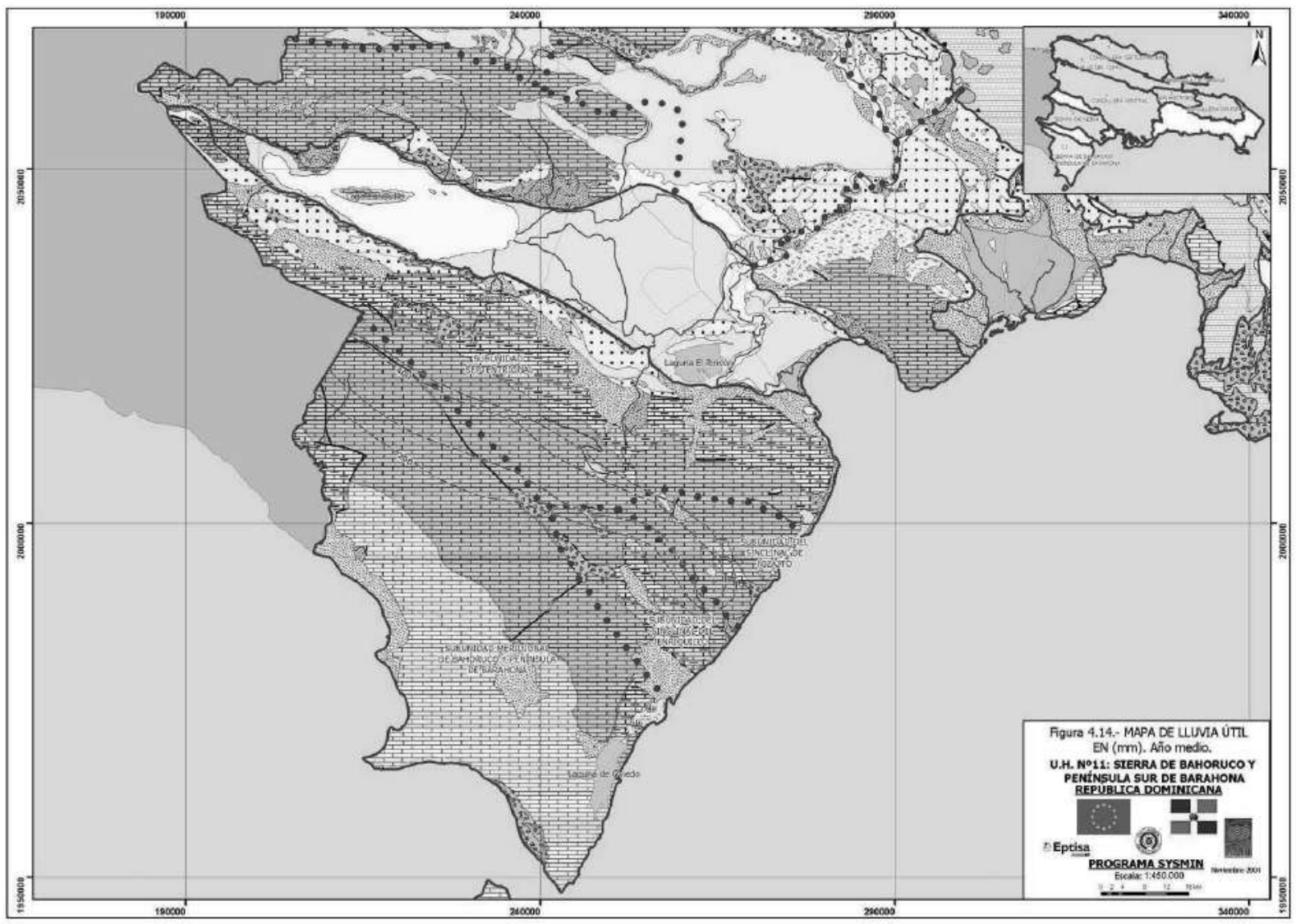

Figura 5. Geología de la región del lago Enriquillo. Fuente: Eptisa (2004). 


\section{Hidrología del Lago Enriquillo}

Aquater (2000) reportó una evaporación de 1547 $\mathrm{mm}$ en Guayabal y Postrer Río. Esto es muy por encima de los $1084 \mathrm{~mm}$ de precipitación durante el 2007, cuando las tormentas Noel y Olga pasaron por la zona del lago Enriquillo. Aparte de la precipitación directa el lago Enriquillo recibe aguas superficiales de ríos y canales desde las partes norte, sur y oeste. Aunque se ha reportado que su cuenca de drenaje incluye 10 sistemas de ríos menores, y que los ríos que se nacen en la sierra de Neyba son perennes, pero la mayoría de estos no drena sus aguas al lago Enriquillo ya que estas son utilizadas para agricultura y ganadería en su mayor parte. Solo dos de estos ríos drenan parte de sus aguas al lago, C-20 y C-21. Fig. 6. Aportando aproximadamente 19, 131,840.00 y 1, 471,680.00 de $\mathrm{M}^{3}$ al año respectivamente. La mayor cantidad de agua superficial que llega al lago es desde la parte sur, donde los canales C-17, C-18 y C-19 Aportan 22, 811,040.00, 22, $495,680.00$ y $15,452,640.00$ de $\mathrm{M}^{3}$ respectivamente. Fig. 6. Desde el Oeste el lago Enriquillo no recibe aguas superficiales, y desde el Este solo en canal Cristóbal, C-11, aporta aproximadamente 26, 490,240.00. Fig. 6 . Se ha podido confirmar que el canal Cristóbal está siendo utilizado mayormente para riego y la mayor parte de sus aguas no llegan al lago Enriquillo. Esto no quiere decir que el mayor aporte de aguas al lago sea desde el Sur, ya que habría que hacer varias pruebas de bombeos para determinar el aporte de las aguas subterráneas al lago desde todos los ángulos. Durante los años 2015 y 2016 se contabilizó un aporte de aguas superficiales de aproximadamente $107,853,120.00 \mathrm{M}^{3}$ por ańo.

La mayoría de los canales que deberían drenar sus aguas al lago Enriquillo no lo hacen, ya que por lo regular son utilizados para riego y permanecen secos prácticamente todo el año. Fig. 7. Ya desde los años 70 estos ríos vienen siendo canalizados para usos de agricultura, cortando su entrada al lago. Incháustegui et al., (1978). Es decir, "El lago Enriquillo tiene una salida, que es evapotranspiración, que va desde $1.200 \mathrm{~mm}$ a $1.800 \mathrm{~mm}$ y durante períodos de sequía prolongados pueden superar la entrada de agua dulce teniendo una evapotranspiración de más de 2.500 mm." González (2015). Buck et al., (2005) calculan la evaporación anual en aproximadamente $2000 \mathrm{~mm}$.

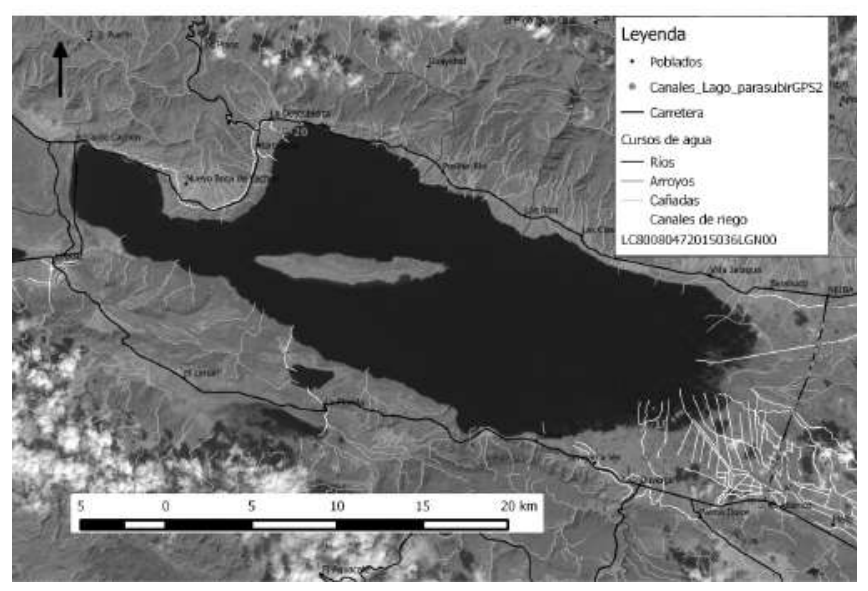

Figura 6. Ríos y Canales alrededor del lago Enriquillo y puntos de muestreo.

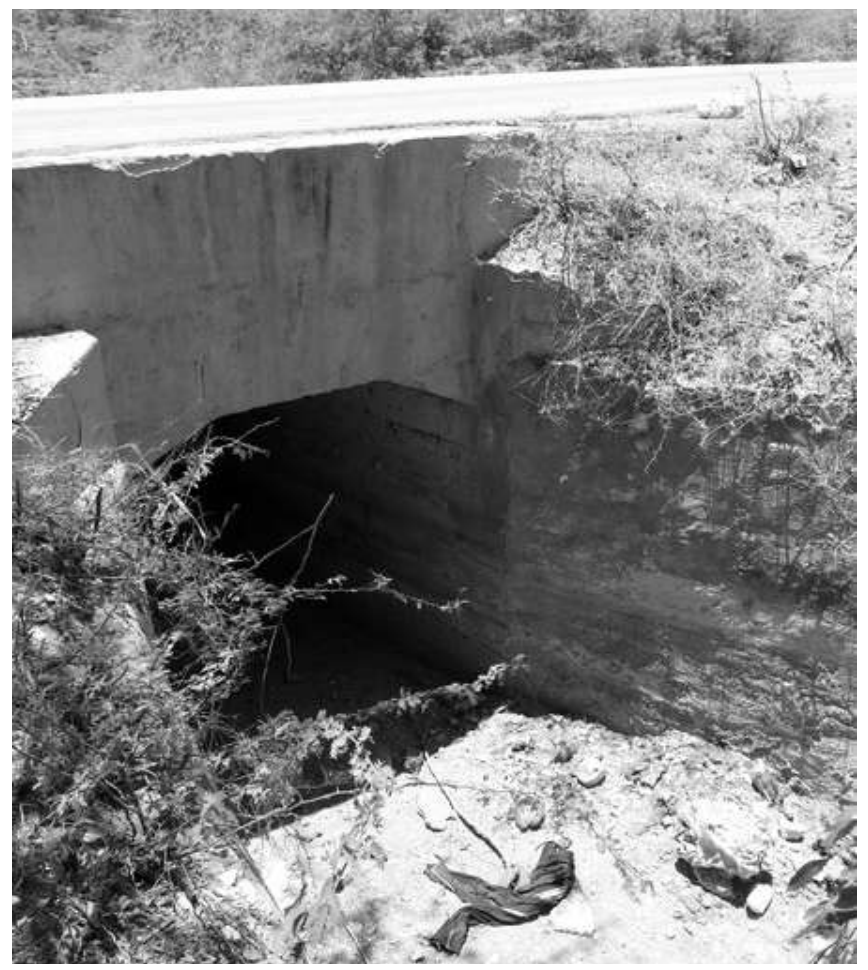

Figura 7. Río seco en la parte este del lago Enriquillo. 
El nivel del agua del lago varía debido a una combinación de eventos de precipitación causados por tormentas y la alta tasa de evaporación de la región, la cual tiene un clima cálido y semiárido donde las. (Cocco Quezada, 2009; González, 2015). La región tiene un clima cálido y semiárido. Las precipitaciones anuales no se distribuyen uniformemente, teniendo precipitaciones máximas en mayo y octubre, aunque esto ha variado durante los últimos ańos. Los meses de diciembre y abril tradicionalmente se han caracterizado por ser secos. (Cocco Quezada, 2009; González, 2015; Vanshan et al., 2015). Esto también ha cambiado durante los últimos años. Durante estos meses la precipitación puede ser menor de $20 \mathrm{~mm}$. Los promedios anuales de precipitación varían en el este y el oeste del lago, teniendo unos $729 \mathrm{~mm}$ en la costa noroeste y unos $508 \mathrm{~mm}$ en el sureste. (Herrera y Orrego, 2011; Vanshan et al., 2015; Méndez-Tejeda et al., 2016). Fig. 8 .

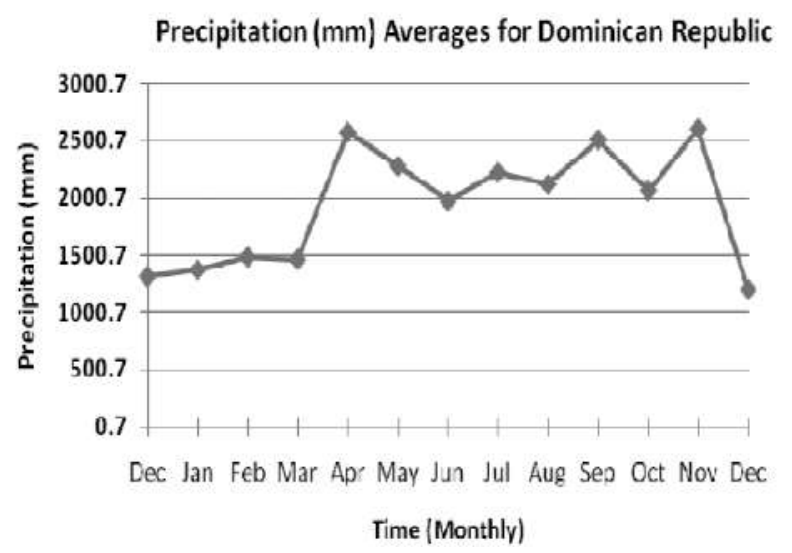

Figura 8. Promedio de precipitación. Lago Enriquillo. Fuente: González, 2010.

Un importante aporte a las aguas del lago Enriquillo viene de la influencia de manantiales, ríos y cañadas que proveen parte de su agua a través del subsuelo. Entre estos están: Boca de Cachón, Las Marías, La Zurza, Las Barías, río Guayabal, río Las Damas, río Bermesí, y río Barreras, contribuyendo al lago aproximadamente 1,100 millones $\mathrm{m}^{3}$ /año (Mann et al., 1991, Gil et al., 2012).

\section{Lago Enriquillo y el crecimiento en el nivel de sus aguas: Hipótesis}

Aunque en los últimos años las publicaciones sobre la crecida del lago Enriquillo han disminuido, aún no se ha llegado a una hipótesis consensuada sobre este fenómeno, aunque la mayoría de los autores reconocen que las tormentas Noel y Olga en el 2007 tuvieron el mayor impacto. El problema es que el lago empezó a crecer a partir de 2004 lo cual generó un debate entre algunos investigadores. Fue durante el mes de mayo de ese mismo año que la provincia Independencia recibió una precipitación de unos $312.6 \mathrm{~mm}$ en 12 horas según datos de la Oficina National de Meteorología (ONAMET). A esto se le conoce como "La tragedia de Jimaní”. No se ha podido localizar ningún estudio posterior a ese evento climático para calcular el balance hídrico del área, para de esa manera determinar la cantidad de agua que se filtró al subsuelo y el tiempo de residencia de la misma. Esto daría una buena idea sobre el impacto de estas precipitaciones en comparación a las tormentas Noel y Olga. Desde el 2004 se propusieron varias hipótesis sobre la crecida del lago Enriquillo, entre ellas: la deforestación (Payano \& Medrano, 2011); eventos meteorológicos (Cocco-Quezada 2009, González 2012 y Schubert 2012); aumento en la sedimentación (Payano \& Medrano, 2011); desvío del río Yaque del Sur (Gil et al., 2012); elevación de los océanos debido al calentamiento global (Herrera \& Orrego, 2011; la proximidad a la Astenósfera (la zona superior del manto terrestre que está inmediatamente debajo de la litosfera), provocando un aumento de la temperatura del agua causando un vapor que tendería a empujar las capas sedimentarias, elevando el nivel del agua o el nivel del fondo de los lagos (Martínez, 2010). Artículos publicados en periódicos locales proponen que la crecida del lago Enriquillo se debe un aumento en los caudales de los ríos que drenan en el lago debido a una mala gestión de infraestructuras hidráulicas. 
Independientemente de las hipótesis planteadas se debe señalar que el lago Enriquillo históricamente ha tenido períodos de crecimiento seguidos de períodos de descenso (Noble 1929, Ducoudray 1981). El período más prolongado de aumento del nivel del lago en el registro histórico fue entre los años 1960 y 1968, cuando los niveles de agua subieron de 45 a $35 \mathrm{mbnm}$, y esto se puede apreciar en el trabajo de Buck et al., (2005), donde se documenta la diferencia del nivel de las aguas del lago entre los años 1950 y 2000. Fig. 9. Muchos investigadores coinciden en el hecho de que los fenómenos meteorológicos extremos que afectaron la región suroeste de la República Dominicana a partir del 2004 han sido responsables, al menos en parte, de la crecida del lago Enriquillo. (Minińo et al., 2015).

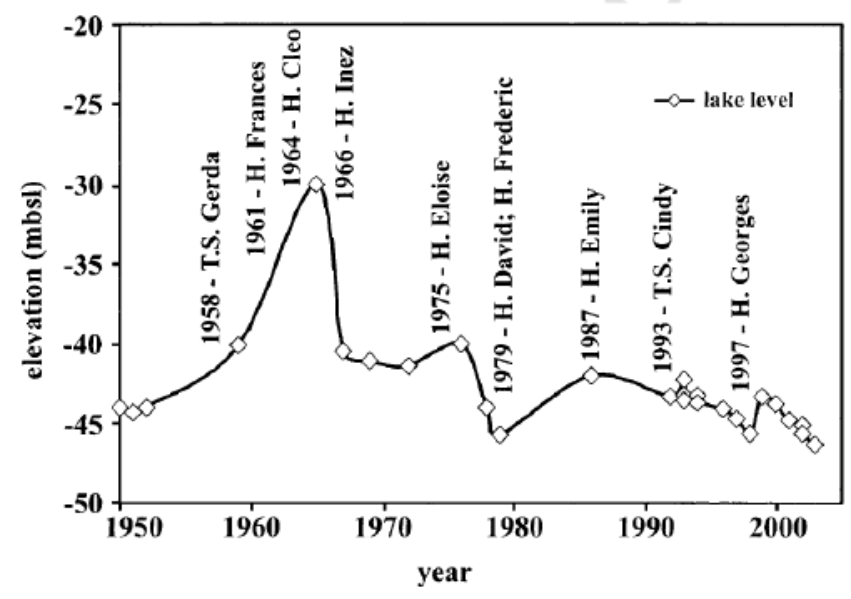

Figura 9. Variación en el nivel de las aguas del lago Enriquillo, 1590-2000. Fuente: Buck et al., 2005.

Imágenes satelitales muestran la variabilidad de la superficie del lago Enriquillo, desde el año 1993, siguiendo el 2002 cuando existía la preocupación de que el lago se secara, hasta el 2016, cuando el lago después de haber tenido su superficie máxima en el año 2013, muestra indicios de decrecimiento. Figs. 10, 11,12 y 13 .

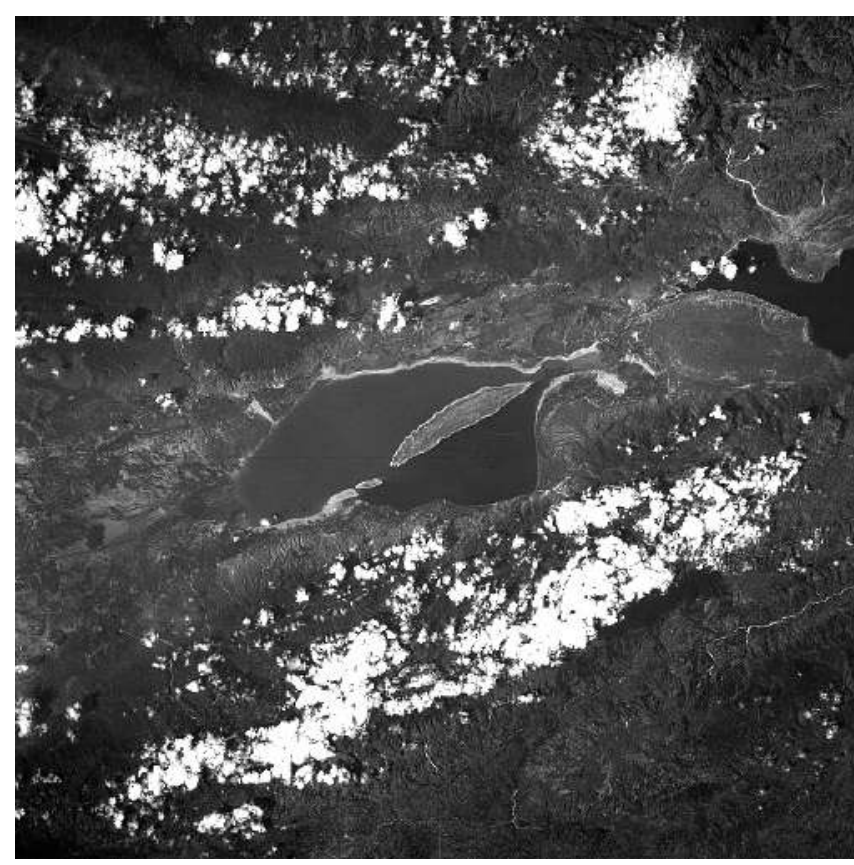

Figura 10. Superficie del lago Enriquillo. Septiembre 1993. Fuente: Wikipedia.

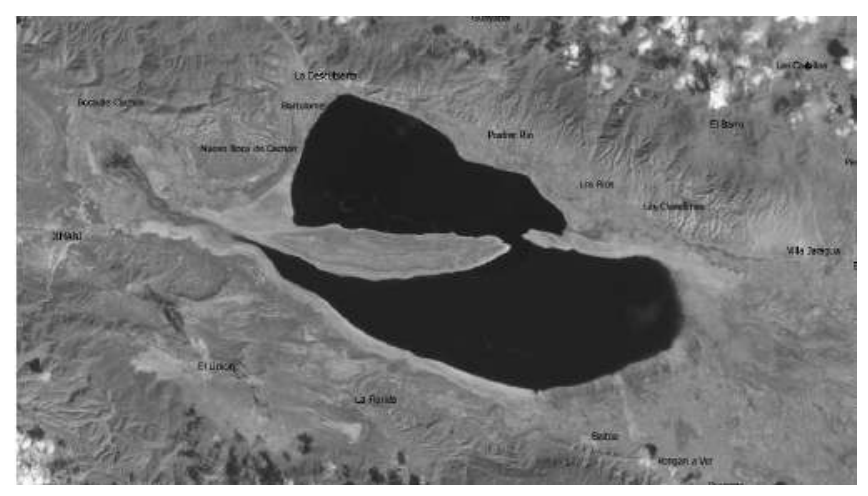

Figura 11. Superficie del lago Enriquillo. 2002. Fuente: Google Earth.

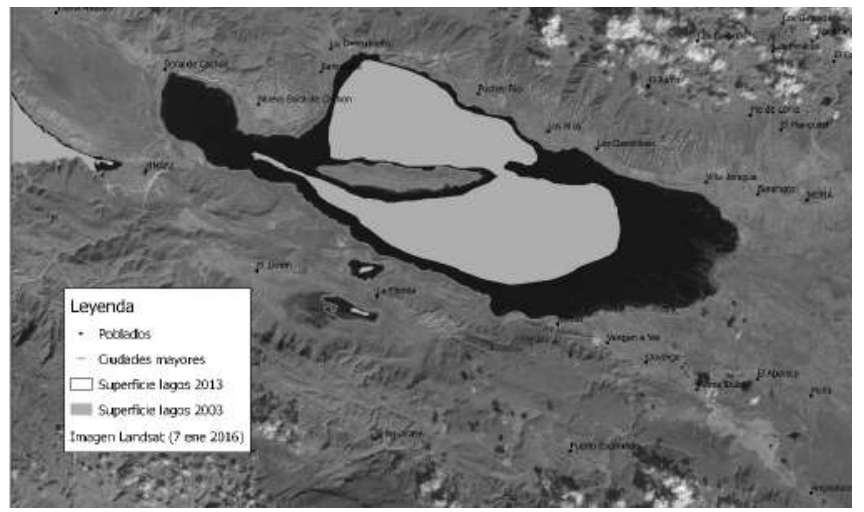

Figura 12. Superficie del lago Enriquillo. 2013. Fuente: Google Earth. 


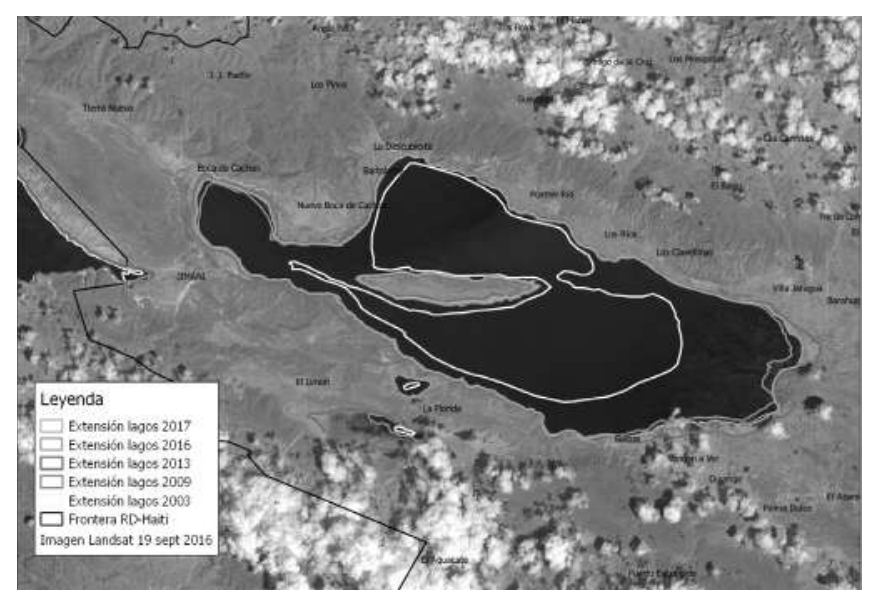

Figura 13. Superficie del lago Enriquillo. 2013-Abril 2017. Fuente: Google Earth.

Jiménez y Galizia (2012) consideran que la tendencia histórica del nivel del lago Enriquillo ha sido disminuir debido a que las aguas que lo alimentan han sido usadas en la agricultura y consumo humano en los pueblos alrededor del lago, mientras que la evaporación es constante. Payano y Medrano (2011) explican que existe una correlación geológica que permite un drenaje, aún por cuantificar, del lago Sumatre o Azuei de Haití hacia el lago Enriquillo. Martínez (2010) considera que un movimiento telúrico podría tener un impacto en el lago Sumatre y verter sus aguas inundando el municipio de Jimaní, lo cual podría prácticamente unir los dos lagos (Sumatre y Enriquillo). CoccoQuezada (2009) también ha expresado lo mismo, pero la topografía del área sugiere que esto es muy poco probable.

\section{Materiales y métodos}

En este proyecto de investigación se utilizó datos tomados del proyecto "Aporte de fuentes de aguas superficiales en la parte Este del Lago Enriquillo y el impacto de esas aguas al crecimiento del lago" el cual fue financiado por los fondos de investigación del Instituto Tecnológico de Santo Domingo (INTEC). Se continuó recolectando datos históricos sobre la hidrología, hidrogeología y geología del área de estudio. Se utilizaron mapas geológicos a escala 1:50,000 para conocer las formaciones y estructuras geológicas, el tipo de rocas alrededor del lago y sus características. También se recopiló datos climáticos del área a través de la National Aeronautics and Space Administration (NASA) y de la European Climate Assessment \& Data, KNMI Climate Explorer.

Se continuó tomando medidas de caudal en la parte oriental del lago Enriquillo, y además se hizo un viaje de reconocimiento alrededor del mismo para localizar los ríos y canales en la parte norte, sur y oeste del lago, e incluirlos en un mapa general de puntos de muestreo. Fig. 6. Se marcaron los las localizaciones de estos ríos y canales utilizando un GPS Garmin GPSmap 62sc.

Una vez localizados todos los ríos y canales a medir se planificaron los viajes de campo a realizar durante los próximos dos años. Se realizaron ocho viajes de campo en las diferentes estaciones del año para medir el flujo de aguas superficiales alrededor del lago Enriquillo utilizando un correntómetro (FP111 Flow Probe, 3'-6'). Fig.14. El flujo o caudal, es la cantidad de agua que pasa a través de una sección del rio o canal por unidad de tiempo. Se calcula multiplicando la velocidad del agua $(\mathrm{m} / \mathrm{s})$ por el área de la sección específica $\left(\mathrm{m}^{2}\right)$, y el resultado es el volumen $\left(\mathrm{m}^{3} / \mathrm{s}\right)$.

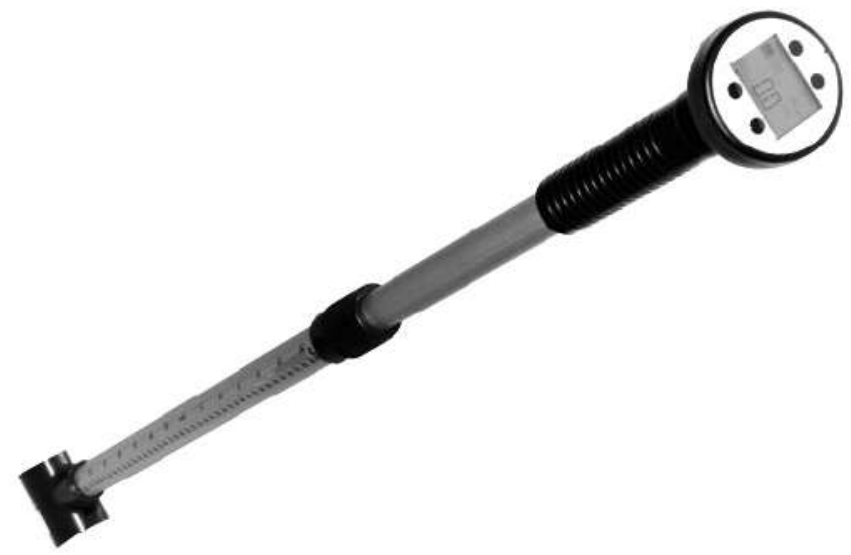

Figura 14. Correntómetro FP311 Global Water Flow Probe. 
Los datos de flujo de caudales, se compararon con otros datos hidrológicos obtenidos a través de ONAMET, el del equipo de investigadores del INTEC y el City College of New York, los dantos de la NASA, y de la European Climate Assessment. Se utilizó el software ArcGIS para la elaboración de mapas temáticos del proyecto de investigación.

En cuanto a la toma de mediciones de los caudales se procedió de la siguiente manera: Se marcó la localización del río o canal utilizando un GPS. Luego se midió el ancho del río utilizando una cinta métrica y de inmediato se midió la profundidad del canal en el centro, en el costado izquierdo y el costado derecho del mismo y se tomó la profundidad promedio para la calculación. Luego se tomaron al menos tres (3) medidas de velocidad del flujo de agua en cada segmento y a diferentes profundidades. Figs. 15, 16 y 17. En algunas ocasiones se tomaron cuatro, cinco y hasta seis mediciones debido a inconsistencias en la lectura del caudalímetro. Se calculó el promedio de la profundidad del canal, así como el promedio de las velocidades de los flujos. Para calcular el área del río se usó la siguiente ecuación:

$$
A=P \times L
$$

Donde $\mathrm{A}=$ Área, $\mathrm{P}=$ Profundidad promedio y $\mathrm{L}$ = Ancho. Para el Canal Cristóbal, el cual es el que aporta mayor agua al lago, la profundidad promedio fue de 0.44 metros, el ancho 10.2 metros, y el área 4.45 metros cuadrados.

Para calcular la descarga total del rio se usó la siguiente ecuación:

$$
\mathrm{Q}=\mathrm{VA}
$$

Donde $\mathrm{Q}=$ Descarga total, $\mathrm{V}=$ Velocidad promedio y A = Área. Para el Canal Cristóbal

$\mathrm{V}=0.26 \mathrm{~m} / \mathrm{s}, \mathrm{A}=4.45 \mathrm{~m}^{2}$ y $\mathrm{Q}=1.15 \mathrm{~m}^{3} / \mathrm{s}$. Estos cálculos se hicieron igual para todos los ríos y canales alrededor del lago Enriquillo.

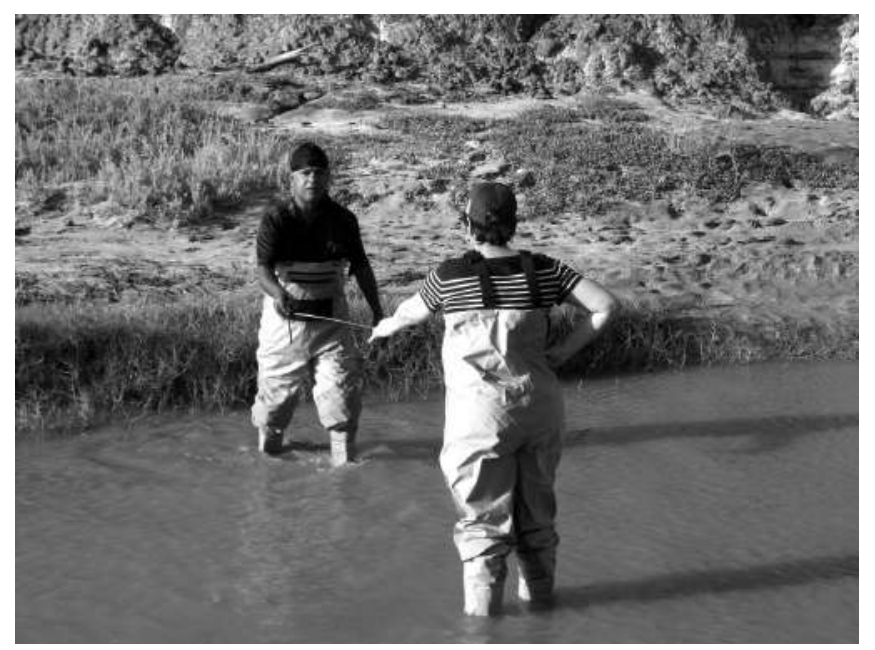

Figura 15. Medición del ancho de río.

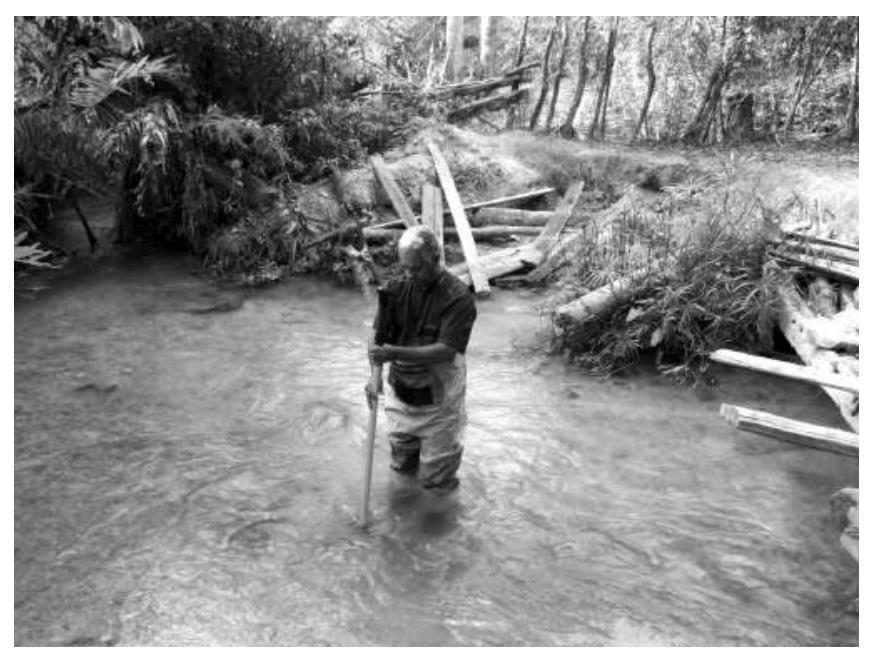

Figura 16. Medición del flujo de río.

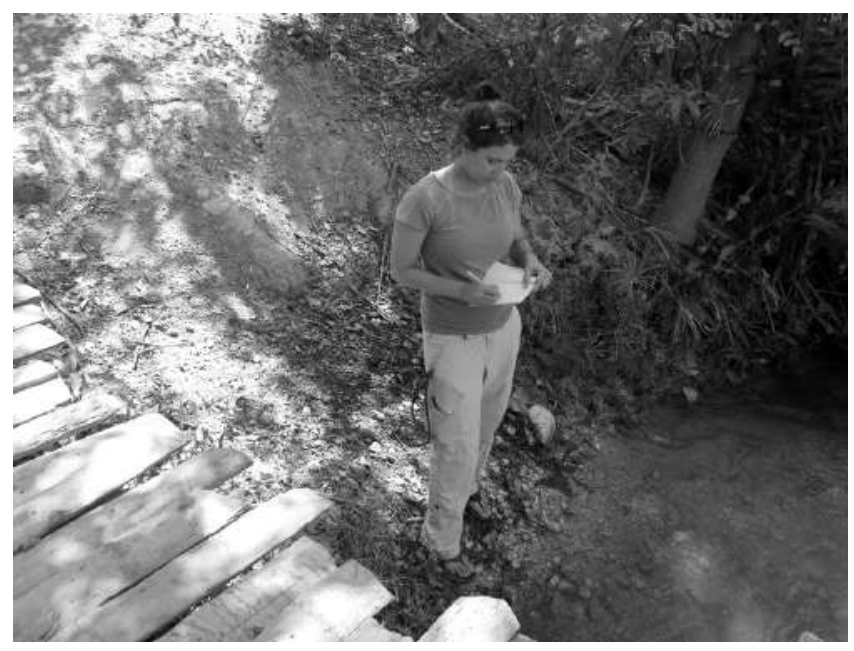

Figura 17. Toma de datos para ser tabulados. 


\section{Discusión}

Mientras que se ha reportado, dentro de los datos escasos para el área de estudio, una evaporación aproximada de $1547 \mathrm{~mm}$ por ańo, la precipitación media es de aproximadamente $900 \mathrm{~mm}$. Fig. 18. La tabla 1 y la figura 19 muestran en la estación de Jimaní una baja en la precipitación a partir del año 2012. El 2016 registró una mayor precipitación que los tres ańos anteriores, pero aún menor que lo que se había registrado hasta el 2012. Cuando se compara esta gráfica con los mapas satelitales se puede observar que el aumento de precipitación del 2016 no fue significativo como para detener el descenso del nivel de las aguas del lago Enriquillo. Esta gráfica también muestra que solo la estación de Jimaní aportó datos de precipitación entre el 1997 y el 2016.

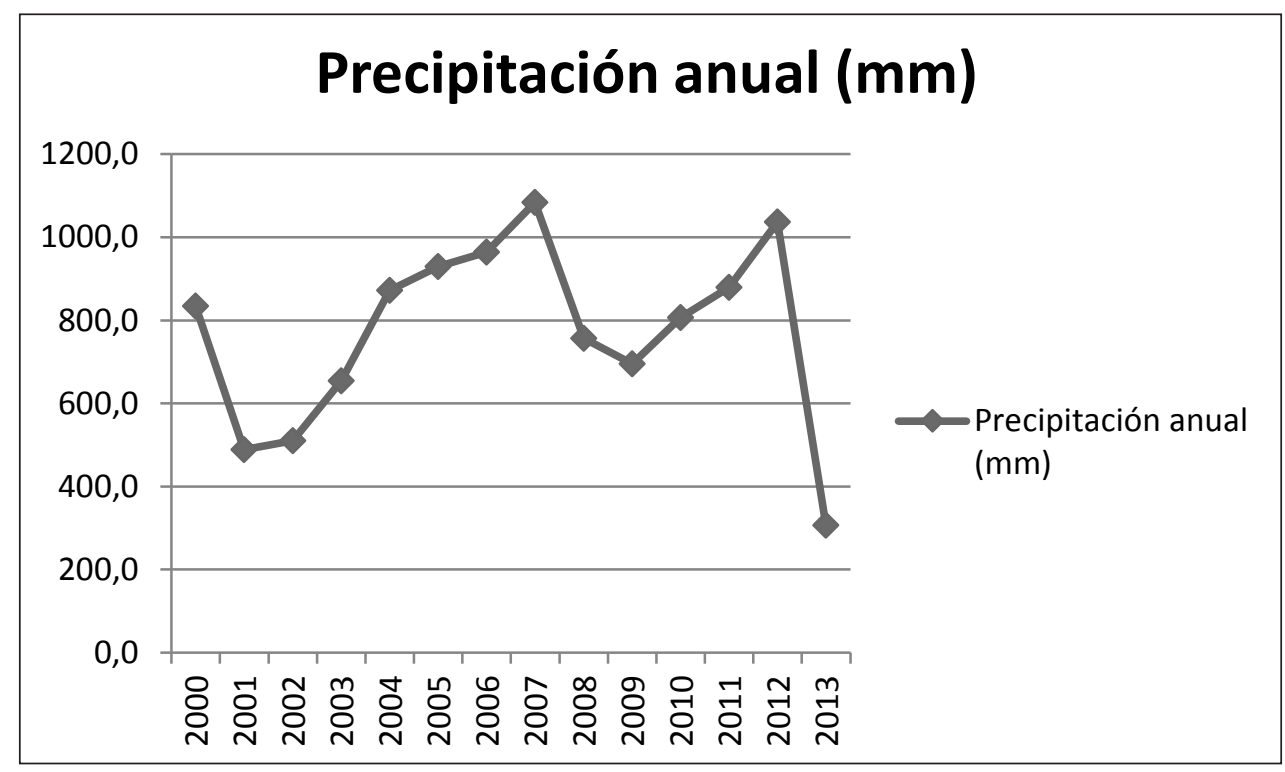

Figura 18 Precipitación anual (mm). Fuente Onamet.

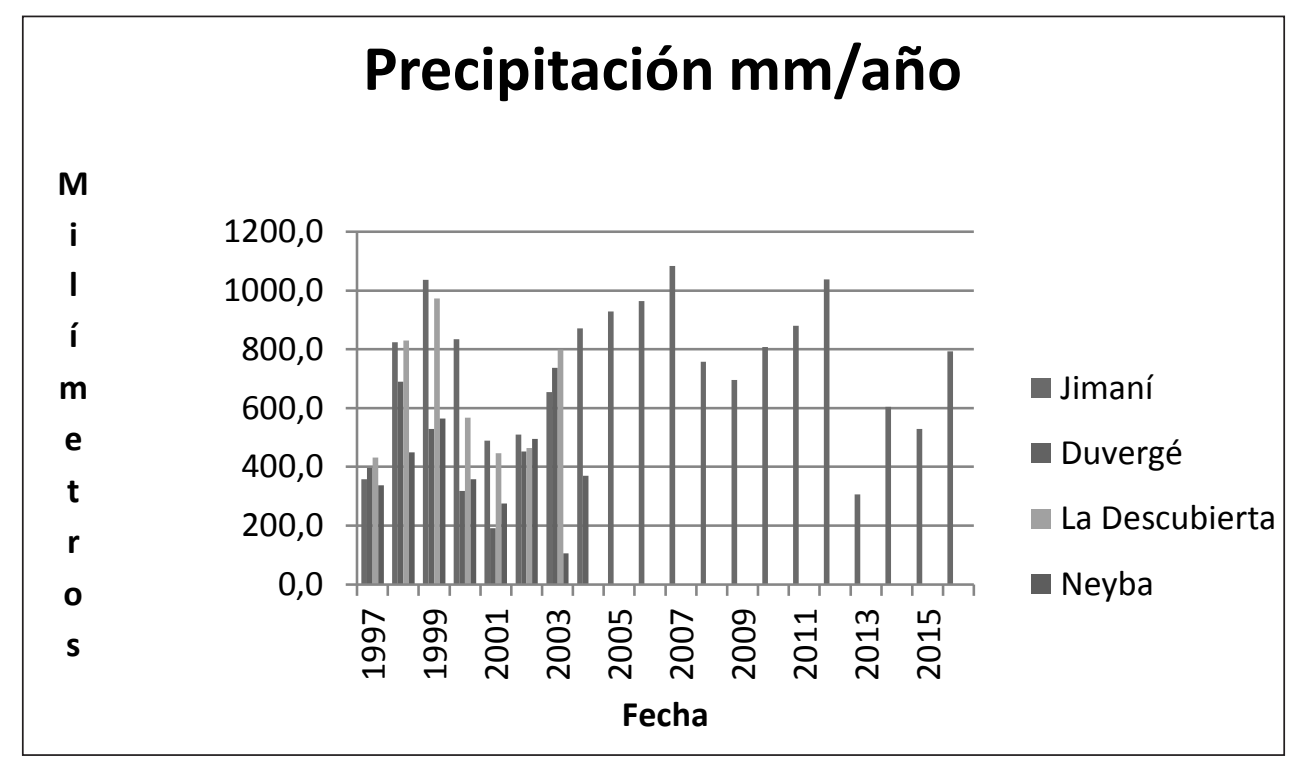

Figura 19. Precipitación en las estaciones de Jimaní, Duvergé, La Descubierta y Neyba. Elaborada con datos de Onamet. 
Tabla 1. Precipitación anual (mm). Estación de Jimaní. Fuente Oficina Nacional de Meteorología.

\begin{tabular}{ccccc}
\hline Año & Jimaní & Duvergé & $\begin{array}{c}\text { La Descu- } \\
\text { bierta }\end{array}$ & Neyba \\
\hline $\mathbf{1 9 9 7}$ & 358.2 & 395.8 & 431 & 337.5 \\
$\mathbf{1 9 9 8}$ & 824.1 & 690.1 & 829.6 & 448.7 \\
$\mathbf{1 9 9 9}$ & 1037.0 & 529.4 & 973.4 & 563.7 \\
$\mathbf{2 0 0 0}$ & 833.9 & 317.8 & 566.7 & 358.1 \\
$\mathbf{2 0 0 1}$ & 488.4 & 191.7 & 446.4 & 275.6 \\
$\mathbf{2 0 0 2}$ & 510.1 & 451.7 & 463.7 & 494.3 \\
$\mathbf{2 0 0 3}$ & 654.7 & 736.6 & 798.7 & 105.6 \\
$\mathbf{2 0 0 4}$ & 871.9 & 368.9 & & \\
$\mathbf{2 0 0 5}$ & 929.3 & & & \\
$\mathbf{2 0 0 6}$ & 963.9 & & & \\
$\mathbf{2 0 0 7}$ & 1084.0 & & & \\
$\mathbf{2 0 0 8}$ & 757.1 & & & \\
$\mathbf{2 0 0 9}$ & 694.9 & & & \\
$\mathbf{2 0 1 0}$ & 807.1 & & & \\
$\mathbf{2 0 1 1}$ & 879.5 & & & \\
$\mathbf{2 0 1 2}$ & 1037.3 & & & \\
$\mathbf{2 0 1 3}$ & 306.3 & & & \\
$\mathbf{2 0 1 4}$ & 604.3 & & \\
$\mathbf{2 0 1 5}$ & 529.1 & & \\
$\mathbf{2 0 1 6}$ & 793.6 & & & \\
\hline
\end{tabular}

Los datos de precipitación de Onamet se compararon con los de la National Aeronautics and Space Administration (NASA) y de la European Climate Assessment \& Data, KNMI Climate Explorer, y se observó que los de Onamet fueron más consistentes, ya que reflejaron datos más precisos durante los años 2004 y 2007 durante las precipitaciones extremas que afectaron la crecida del lago Enriquillo.

\section{Resultados}

Durante los años 2015 y 2016 se localizaron 21 fuentes de aguas superficiales alrededor del lago Enriquillo. De estas solo 8 tuvieron caudal durante este período. De los 8 con caudales solo 6 drenan al lago, mientras que los otros 2 quedan secos al ser sus aguas utilizadas para riego. Las 6 fuentes de agua superficial que se contabilizaron aportaron aproximadamente 107 , $853,120.00 \mathrm{M}^{3}$ por año. De estas, el canal Cristóbal fue el que mayor aporte hizo al lago con una descarga de aproximada de 26, 490,240.00 $\mathrm{M}^{3}$ por ańo.

Aunque a mediados y finales del 2016 se registraron precipitaciones mayores que los meses anteriores, la mayoría de los canales no registraron cauce, lo cual sugiere que mucho antes de llegar al lago estas aguas son utilizadas para riego.

\section{Conclusión}

La crecida del lago Enriquillo fue causada por precipitación extrema durante el período 20042007, y seguida por altas precipitaciones dentro de lo normal para el área durante los años 20082011. A partir del 2013 el lago empezó a mostrar un descenso continuo en el nivel de sus aguas, lo cual va acorde con una disminución de la precipitación. Al momento existe una tendencia a la disminución de aguas superficiales que drenen en el lago Enriquillo, debido a que durante los últimos ańos se ha incrementado el uso de estas para agricultura, además de que la creciente población también tendrá un impacto negativo en la cantidad de aguas superficiales que llegan hasta el lago. Para poder tener un buen manejo de los datos hidrológicos que puedan predecir con más precisión el nivel de las aguas del lago Enriquillo es necesario hacer ensayos de bombeo en los acuíferos alrededor del mismo.

\section{Bibliografía}

Aquater. (2000). Estudio Hidrogeológico del Valle de Neiba. Memorias Finales. 154 pp.

Buck, D. G., Brenner, M., Hodell, D. A., Curtis, J. H., Martin, J. B. \& Pagani, M. (2005). Physical and chemical properties of hypersaline Enriquillo Lake, Dominican Republic. Verh. Internat. Verein. Limnol. 29. Stuttgart.

Coco Quezada, A. (2017). El ciclo hidrológico del lago Enriquillo y la crecida extrema del 2009. http://www.acqweather.com/Avalancha Jimani. 
html. República Dominicana, Abril 16, 2009. Bajado el 15 de marzo del 2017.

Dolan, J., Mann, P., De Zoeten, R., Heubeck, C., Shirona, J. \& Monechi, S. (1991). Sedimentologic, stratigraphic, and tectonic synthesis of EoceneMiocene Sedimentary Basins, Hispaniola and Puerto Rico. Geological Society of America. Special Paper 262, Boulder, Colorado.

Ducoudray, F. S. (1981). Y una escalera grande para bajar al lago. En: Incháustegui A., DelgadoMalagón, B (eds) 2006. La naturaleza dominicana: artículos publicados en el suplemento sabatino del periódico El Caribe, 1978-1989 / Felix Servio Ducoudray Santo Domingo: Grupo León Jimenes. 6 v. :il.- (Colección Centenario Grupo León Jimenez).

Eptisa Group. (2004). Programa Sysmin. Estudio hidrogeológico nacional de la República Dominicana Fase II. Volumen 10: Resumen, conclusiones y recomendaciones. Memoria General.

Gil Pichardo, F., Lo Conte, L. \& Regio, G. (2012). Alternativas productivas a mediano y largo plazo para las familias afectadas por la crecida del nivel del lago Enriquillo. Infome. OXFAM-ITALIA, Proyecto Apoyo de emergencia para restaurar los medios de vida de los agricultores afectados por la crecida del lago Enriquillo.

González, J. (2015). Growth of Lago Enriquillo. Final Report. Department of Environmental Engineering. The City College of New York.

Herrera, M. A. \& Orrego Campo, J. C. (2011). Revisión del Estado de la Situacion de Riesgo Climatico y su Gestión en República Dominicana. IISD Reporte.

Incháustegui, S. J., Gutiérrez W., Rivas V., Álvarez V., Núñez de Ricart N., Bonnelly de Calventi, I. (1977). Notas sobre la ecología del lago enriquillo República Dominicana en 1977. En Bonnelly de Calventi (Eds.) 1978. CIBIMA: Conservación y Ecodesarrollo. Universidad Autónoma de Santo Domingo. Santo Domingo. Editora Alfa y Omega.

Jiménez Cisneros, B. \& Galizia Tundisi, J. (2012). Diagnóstico del agua en la Américas. Red interamericana de academias de ciencias. Foro consultivo científico y tecnológico. Santo Domingo.

Koehler, R. D., Mann, P. \& Brown, L. A. (2009). Tectonic geomorphology and paleoseismology of strike-slip faults in Jamaica: Implications for distribution of strain and seismic hazard along the southern edge of the Gonave microplate. Eos Trans. AGU. Fall Meeting. G33B-0658.

Mann, P., McLaughlin, P. P., \& Cooper, C. (1991). Geology of the Azua and Enriquillo basins, Dominican Republic; 1 Structure and tectonics". Geological society of America Special Paper 262, Boulder, Colorado.

Mann, P., Taylor, F., Edwards, R. \& Ku, T. (1995). Actively evolving microplate formation by oblique collision and sideways motion along strike-slip faults: An example from the northeastern Caribbean plate margin. Tectonophysics 246: 1-69.

Mann, P., Calais, E., Demets, C., Prentice, Carol S., \& Wiggins-Grandison, M. (2008). EntiquilloPlantain Garden Strike-Slip Fault Zone: A Major Seismic Hazard Affecting Dominican Republic, Haiti and Jamaica". 18th Caribbean Geological Conference.

Martínez, H. (2010). Consideraciones acerca del riesgo de inundación del poblado de Jimaní. Riesgo de Inundación debido al rebose del lago Saumätre o Azuei hacia el Lago Enriquillo. IEM 22065.

Méndez-Tejeda, R., Rosado, G., Rivas, V., Montilla, T., Hernández, S., Ortíz, A., Santos, F. (2016). Climate Variability and Its Effects on the Increased Level of Lake Enriquillo in the Dominican Republic, 2000-2013. Applied Ecology and Environmental Sciences 4 (1): 26-36. 
Miniño Mejía, V., León, Y., Quintana, C. \& Rosario, A. (2015). Aporte de fuentes de aguas superficiales en la parte Este del Lago Enriquillo y el impacto de esas aguas al crecimiento del lago. Ciencia y Sociedad 40(2): 425-448.

Payano O. \& Medrano, R. (2011). Análisis de la situación actual del lago Enriquillo de la República Dominicana. Instituto IMDEA Agua.

Noble G. K. (1929). Trailing the rhinoceros iguana. Natural History 23 (6): 541-558

Romero Luna, E. \& Poteau, A. (2011). Water Level Fluctuations of Lake Enriquillo and Lake Saumatre in Response to Environmental Changes. A Masters of Engineering Project Presented to the Faculty of the Graduate School of Cornell University In Partial Fulfillment of the Requirements for the Degree of Master of Engineering.

Vanshan, D., Wright, M., Hornbach, C., Mchugh, P. (2015). Factors Contributing to the 2005-Present, Rapid Rise in Lake Levels, Dominican Republic and Haiti (Hispaniola). Natural Resources 6: 465-481. 